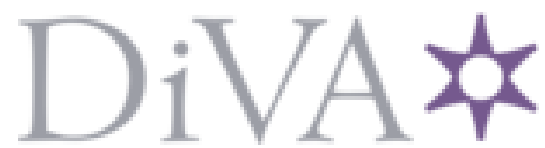

http://www.diva-portal.org

This is the published version of a paper published in Clinical Physiology and Functional Imaging.

Citation for the original published paper (version of record):

Rosendahl, L., Ahlander, B-M., Björklund, P-G., Blomstrand, P., Brudin, L. et al. (2010) Image quality and myocardial scar size determined with magnetic resonance imaging in patients with permanent atrial fibrillation: a comparison of two imaging protocols Clinical Physiology and Functional Imaging, 30(2): 122-129 https://doi.org/10.1111/j.1475-097X.2009.00914.x

Access to the published version may require subscription.

N.B. When citing this work, cite the original published paper.

Permanent link to this version:

http://urn.kb.se/resolve?urn=urn:nbn:se:oru:diva-72074 


\title{
Image quality and myocardial scar size determined with magnetic resonance imaging in patients with permanent atrial fibrillation: a comparison of two imaging protocols
}

Lene Rosendahl ${ }^{1,2}$, Britt-Marie Ahlander ${ }^{3}$, Per-Gunnar Björklund ${ }^{1}$, Peter Blomstrand ${ }^{1}$, Lars Brudin ${ }^{4,5}$ and Jan E. Engvall ${ }^{2,5}$

\begin{abstract}
${ }^{1}$ Department of Clinical Physiology, Ryhov County Hospital, Jönköping, ${ }^{2}$ Center for Medical Image Science and Visualization, Linköping University, Linköping, ${ }^{3}$ Department of Radiology, Ryhov County Hospital, Jönköping, ${ }^{4}$ Department of Clinical Physiology, Kalmar County Hospital, Kalmar, and ${ }^{5}$ Department of Medical and Health Sciences, Linköping University, Linköping, Sweden
\end{abstract}

\section{Summary}

\section{Correspondence}

Lene Rosendahl, Department of Clinical Physiology, Ryhov County Hospital, 55185 Jönköping, Sweden E-mail: lene.rosendahl@li.se

\section{Accepted for publication}

Received 7 July 2009;

accepted 18 November 2009

\section{Key words}

atrial fibrillation; magnetic resonance imaging; myocardial infarction; segmented inversion recovery 2D fast gradient echo; single shot inversion recovery 2D steady-state free precession
Background: Magnetic resonance imaging (MRI) of the heart generally requires breath holding and a regular rhythm. Single shot 2D steady-state free precession (SS_SSFP) is a fast sequence insensitive to arrhythmia as well as breath holding. Our purpose was to determine image quality, signal-to-noise (SNR) and contrast-to-noise (CNR) ratios and infarct size with a fast single shot and a standard segmented MRI sequence in patients with permanent atrial fibrillation and chronic myocardial infarction.

Methods: Twenty patients with chronic myocardial infarction and ongoing atrial fibrillation were examined with inversion recovery SS_SSFP and segmented inversion recovery 2D fast gradient echo (IR_FGRE). Image quality was assessed in four categories: delineation of infarcted and non-infarcted myocardium, occurrence of artefacts and overall image quality. SNR and CNR were calculated. Myocardial volume $(\mathrm{ml})$ and infarct size, expressed as volume $(\mathrm{ml})$ and extent $(\%)$, were calculated, and the methodological error was assessed.

Results: SS_SSFP had significantly better quality scores in all categories $(P=0.037$, $P=0.014, P=0.021, P=0.03) . S N R_{\text {infarct }}$ and $S_{N N R}$ blood were significantly better for IR_FGRE than for SS_SSFP $(P=0 \cdot 048, P=0 \cdot 018)$. No significant difference was found in $\mathrm{SNR}_{\text {myocardium }}$ and CNR. The myocardial volume was significantly larger with SS_SSFP $(170 \cdot 7$ versus $159 \cdot 2 \mathrm{ml}, \mathrm{P}<0 \cdot 001)$, but no significant difference was found in infarct volume and infarct extent.

Conclusion: SS_SSFP displayed significantly better image quality than IR_FGRE. The infarct size and the error in its determination were equal for both sequences, and the examination time was shorter with SS_SSFP.

\section{Introduction}

The detection of myocardial scar with late gadolinium enhancement (LGE) magnetic resonance imaging (MRI) is evolving as standard work-up postmyocardial infarction and is also used for the investigation of the aetiology of heart failure (Kim et al., 1999). Attractive features of LGE are a high reproducibility (Mahrholdt et al., 2002) and a high spatial resolution which enables the determination of the transmurality of a myocardial infarction, a feature that is related to the likelihood of recovery after revascularization (Hillenbrand et al., 2000; Kim et al., 2000; Kitagawa et al., 2003).

The quest for better image quality is constantly driving imaging to improved performance. However, all parameters cannot be optimized simultaneously and compromises are always necessary. In MRI, a regular cardiac rhythm has been a prerequisite for producing high quality images. In the older patient population, arrhythmia as well as difficulty in breath holding because of heart failure or obstructive lung disease is frequent and patients are sometimes simply uncooperative. Because of the ageing population in most countries, these problems are continuously increasing.

We have used a prospectively ECG-gated, segmented inversion recovery $2 \mathrm{D}$ fast gradient echo (segmented turboFLASH according to the vendor, but here abbreviated IR_FGRE) as the reference sequence to which other techniques have been compared (Kim et al., 2000; Simonetti et al., 2001). IR_FGRE works best in patients in regular sinus rhythm. Using ECG- 
gating, a time delay forces the acquisition to diastole where the motion of the heart is minimal. On our scanner system, a segmented acquisition requires one breath hold per slice. Because complete coverage of the heart in the short-axis view, depending on slice thickness, requires 10-12 slices, successful breath holding may be difficult to achieve for some patients.

Single shot inversion recovery 2D steady-state free precession (single shot trueFISP, here abbreviated SS_SSFP) is a fast technique that acquires one slice during one heart beat. The single shot technique should be able to reduce the negative effect of arrhythmias. Artefacts from cardiac motion and respiration should be less frequent compared with a segmented pulse sequence technique at a cost of a lower spatial resolution.

The steady-state free precession techniques in general offer high contrast-to-noise ratio (CNR) between myocardium and blood at a high signal-to-noise ratio (SNR) (Thiele et al., 2001) which may facilitate volumetric measurements of the left ventricle and reduce observer dependence (Plein et al., 2001). In a comparison of cine_SSFP with fast gradient echo in the assessment of ventricular function, cine_SSFP allowed better detection of the endocardial border (Barkhausen et al., 2001). Because of the relatively high SNR and CNR of the SSFP sequence in general, a single shot version might be suitable for the detection of myocardial scar. It has been shown that SS_SSFP provides adequate image quality compared with IR_FGRE (Li et al., 2004). There is a close correlation between the two sequences in assessing infarct volume in patients with sinus rhythm (Huber et al., 2004, 2006). To our knowledge, no one has so far compared the performance of the two sequences in patients with atrial fibrillation.

The purpose of this study was to determine image quality of the two scar sequences as well as for cine imaging in patients with permanent atrial fibrillation and previous myocardial infarction. An additional aim was to investigate whether a potential difference in image quality would influence infarct sizing. We also intended to compare the signal intensity (SI), SNR and CNR achieved. Because cine-MRI only provided a single averaged heart beat, wall motion and global systolic performance was determined with echocardiography (3 s loops). In addition, the time required for the application of either of the two sequences was measured.

\section{Methods}

\section{Study population}

Twenty patients, all men, age $75 \pm 6$ years (range 59-83) were enroled in the study, for details see Table 1. All 20 patients had myocardial infarction determined from chest pain, ECG abnormalities and/or elevated levels of either Troponin $\mathrm{T}$ $>0.05 \mu \mathrm{g} \mathrm{l}^{-1}$ or creatine kinase $\mathrm{MB}>5.0 \mu \mathrm{gl}^{-1}$, in at least two blood samples while hospitalized. The diagnosis had to be confirmed more than 6 weeks prior to the MRI examination. Five of the patients enroled had a repeat myocardial infarction, the others had first time infarcts. Two of the patients had (C) 2009 The Authors

Journal compilation (c) 2009 Scandinavian Society of Clinical Physiology and Nuclear Medicine 30, 2, 122-129
Table 1 Patient characteristics and number of patients with medication.

Age 75 (Range 59-83)

Number of males

HR (mean $\pm \mathrm{SD})$ $71 \pm 19$

LVEF (mean \% \pm SD) $39 \cdot 3 \pm 8 \cdot 6$

Anticoagulants

Betablockers

Other antihypertensives

Statins

Oral hypoglycemic agents

HR, heart rate; LVEF, left ventricular ejection fraction.

previous surgical revascularization. At the time of the MRI study, all patients were in atrial fibrillation. Exclusion criteria were contraindications for MRI such as an implantable cardiac device, ferromagnetic clips and claustrophobia. A glomerular filtration rate exceeding $30 \mathrm{ml} \mathrm{min}^{-1}$ per $1.73 \mathrm{~m}^{2}$ was required for the use of gadolinium-containing MRI contrast. No patient was excluded because of technical failure or poor image quality. The study was approved by the Regional ethical review board in Linköping and complied with the Declaration of Helsinki. All patients gave informed consent.

\section{Magnetic resonance imaging}

The patients were examined in a $1.5 \mathrm{~T}$ Siemens Symphony scanner (Siemens, Erlangen, Germany) in supine position. A circular polarized body-array surface coil was used. Heart rate and rhythm was monitored from the ECG. A cannula was placed in the antecubital vein for the injection of contrast material. Gadopentetate dimeglumine, $0 \cdot 2 \mathrm{mmol} \mathrm{kg}{ }^{-1}$ bodyweight, max dose $30 \mathrm{ml}$ (Schering Nordiska $\mathrm{AB}$, Järfälla, Sweden) was administered in all patients. Scout images were obtained to locate the heart. The following standard views were used for LGE as well as cine images: three long axis slices (two-, three- and four-chamber views) and as many short-axis slices as necessary to cover the entire left ventricle, on average ten slices (range 8-12). The cine images had the following technical data: repetition time (TR) $43 \mathrm{~ms}$, echo time (TE) $1.3 \mathrm{~ms}$, flip angle (FA) $72^{\circ}$, slice thickness $8 \mathrm{~mm}$, matrix $192 \times 156$, field-of-view (FOV) $320 \mathrm{~mm}$. LGE imaging started $10 \mathrm{~min}$ after contrast administration. For LGE images, two sequences were used in the standard positions, a single shot (SS_SSFP) sequence, and for comparison a segmented (IR_FGRE) sequence (Kim et al., 2000; Simonetti et al., 2001). For the single shot sequence, the following settings were used: $\mathrm{TR}=10.8 \mathrm{~ms}, \mathrm{TE}=1.26 \mathrm{~ms}, \mathrm{FA}=50^{\circ}$, bandwidth $(\mathrm{BW})=1180 \mathrm{~Hz}$, slice thickness $=8 \mathrm{~mm}$, image matrix $=$ $192 \times 108$, number of excitations $($ NEX) $=1$, FOV $=380 \mathrm{~mm}$. Corresponding settings for the segmented sequence were: $\mathrm{TR}=12 \mathrm{~ms}, \quad \mathrm{TE}=5.4 \mathrm{~ms}, \quad \mathrm{FA}=30^{\circ}, \quad \mathrm{BW}=140 \mathrm{~Hz}$, slice thickness $=8 \mathrm{~mm}$, image matrix $=256 \times 160$, NEX $=1$ and $\mathrm{FOV}=380 \mathrm{~mm}$. The segmented acquisition required 12 heart beats per breath hold, was triggered on the R-wave of the ECG 
and acquired 25 phase encoding lines every other heart beat. A 300-ms time delay was added to force the acquisition to the diastolic phase where the movement of the heart is minimal ( $\mathrm{Lu}$ et al., 2001). However, because the patients had irregular R-R interval on the ECG, the actual timing of the acquisition differed between beats. In the single shot acquisition, breath holding was not required. Instead, the acquisition was initiated by the technician from end-expiration in the respiratory trace. In the segmented cine imaging, the patients were instructed to hold their breath in end-expiration. For both sequences, the optimal inversion time was chosen from a midventricular short-axis slice where the signal from healthy, normal myocardium (myo) was nulled (Simonetti et al., 2001; Gupta et al., 2004).

\section{Magnetic resonance image analysis}

Visual subjective quality assessment

Visual assessment of the quality of the LGE images was performed in view of the following four aspects: delineation of (i) noninfarcted and (ii) infarcted myocardium (inf), (iii) the occurrence of motion- and other artefacts and (iv) an overall evaluation of the left ventricle. A five-point rating scale was used for all groups with five as the highest and one as the lowest grade (Bongartz et al., 2000). A score of 5 was given when the parameters were considered 'very good'. A score of 4-3-2 and 1 was given when the parameter (segmentation of non-infarcted- and inf, artefacts and overall impression) was assessed as 'good', 'moderate', 'poor' and 'very poor', respectively. For visual assessment of the quality of the cine images, each short-axis slice of the left ventricle was evaluated in two aspects: (i) the occurrence of artefacts and (ii) overall evaluation of the motion (ghosting, blurring and irregularity) of the left ventricular wall, using ' 5 ' as the highest grade and ' 1 ' as the lowest grade. The quality of the LGE- and cine images was evaluated by two observers and averaged. The two observers were blinded as to the sequence information except for cine.

\section{Volume measures}

Segmentation of the endo- and epicardium of the left ventricle as well as the scar was performed by two observers using a semiautomatic software, 'Segment', (Heiberg et al., 2008). The papillary muscles were included in the myocardial mass/infarction if they were attached to the myocardium in that particular slice. Left ventricular myocardial muscle volume, scar and enddiastolic volume were calculated. The infarct size was expressed in terms of volume $(\mathrm{ml})$ and extent (related to the total volume of the myocardium in \%). All measurements were averaged from the two observers.

\section{Signal-to-noise ratio and contrast-to-noise-ratio}

The SI, the SNR and the CNR values were determined on images from both sequence types. To calculate the SI, regions of interest (ROIs), 25-50 $\mathrm{mm}^{2}$, were placed in the myo and in the inf. In the blood pool (bp), a larger ROI of at least $300 \mathrm{~mm}^{2}$ was drawn. Noise was defined as the standard deviation of the SI measurement in the air outside the patient. The SNR for different cardiac regions was calculated by dividing each SI by the noise. The CNR value for the inf in comparison to the myo was calculated as follows: CNR: $\left(\mathrm{SI}_{\mathrm{inf}}-\mathrm{SI}_{\mathrm{myo}}\right) /$ noise. The CNR value for the inf in comparison with the blood in the ventricular cavity was calculated as follows: $\mathrm{CNR}$ : $\left(\mathrm{SI}_{\mathrm{inf}}-\mathrm{SI}_{\mathrm{bp}}\right) /$ noise.

\section{Echocardiography}

Left ventricular function was determined with echocardiography (Siemens Sequoia 256 or 512; Siemens Healthcare Inc, Mountainview, CA, USA) on the same day as the MRI examination. The patients were investigated in the left lateral position, with cine-loop grey scale recordings from the apical and parasternal positions. Cine-loops were stored digitally for later review. The left ventricle was divided into 16 segments (Schiller et al., 1989). Wall motion was determined by two independent observers and the mean value of each segment was used. Wall motion scoring used conventional steps such as: normal $=1$, hypokinetic $=2$, akinetic $=3, \quad$ dyskinetic $=4$, aneurysm $=5$. Scores were summed and divided by the 16 segments to obtain a global wall motion score index (WMSI) for the left ventricle for each patient. Left ventricular ejection fraction was calculated using the biplane Simpson's method of discs and averaged from two consecutive heart beats.

\section{Statistics}

For image quality, Wilcoxon pairwise signed rank test was used to compare modality performance based on the ordinal-scaled criteria. Interobserver variability (two raters) was expressed as standard error of a single determination ( $S_{\text {method}}$ ) using the formula, first proposed by Dahlberg (1940): $S_{\text {method }}=\sqrt{\left(\sum \mathrm{d}_{\mathrm{i}}^{2} /(2 \mathrm{n})\right)}$, where $\mathrm{d}_{\mathrm{i}}$ is the difference between the $\mathrm{i}$ :th paired measurement and $\mathrm{n}$ is the number of differences. $S_{\text {method }}$ was also expressed as $\%$ over all means. F-test was used to compare

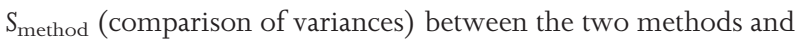
kappa statistics was used to evaluate interobserver agreement.

The volume measures were reasonable well normally distributed and the difference between the two methods (IR_FGRE versus SS_SSFP) was analysed by two-sided t-test for paired observations. Correlation coefficients and related $\mathrm{p}$ values are reported, Bland-Altman plots used and slope differences analysed by t-test. Analyses were performed using SPSS 13.0 (SPSS Inc, Chicago, IL, USA). Two-tailed P-values were used with $\mathrm{P} \leq 0.05$ considered to indicate statistical significance.

\section{Results}

\section{Quality assessment}

SS_SSFP displayed significantly better image quality than IR_FGRE in all four aspects assessed, Table 2. Also, artefacts 
Table 2 Image quality levels are used in the assessment of quality. Mean values from two interpreters. Statistically significant differences are bolded. Wilcoxon pairwise signed rank test was used.

\begin{tabular}{|c|c|c|c|c|c|}
\hline \multirow[b]{2}{*}{ Quality assessment } & \multicolumn{2}{|r|}{ IR_FGRE } & \multicolumn{2}{|r|}{ SS_SSFP } & \multirow[b]{2}{*}{$P$-value } \\
\hline & Mean (SD) & Median (quartile range) & Mean (SD) & Median (quartile range) & \\
\hline Non-infarcted myocardium delineation & $3 \cdot 6(0 \cdot 7)$ & $3 \cdot 4(3 \cdot 1-4 \cdot 2)$ & $3 \cdot 9(0 \cdot 4)$ & $4 \cdot 0(3 \cdot 6-4 \cdot 2)$ & 0.037 \\
\hline Infarcted myocardium delineation & $2 \cdot 7(0 \cdot 8)$ & $2 \cdot 7(2 \cdot 1-3 \cdot 3)$ & $3 \cdot 2(0 \cdot 6)$ & $3 \cdot 1(2 \cdot 8-3 \cdot 6)$ & 0.014 \\
\hline Occurrence of artefacts & $4 \cdot 0(0 \cdot 8)$ & $4 \cdot 1(3 \cdot 6-4 \cdot 8)$ & $4.5(0 \cdot 4)$ & $4 \cdot 6(4 \cdot 4-4 \cdot 9)$ & 0.021 \\
\hline Overall assessment of left ventricle/left ventricle motion & $3 \cdot 3(0 \cdot 7)$ & $3 \cdot 3(2 \cdot 9-3 \cdot 9)$ & $3 \cdot 8(0 \cdot 4)$ & $3 \cdot 8(3 \cdot 5-4 \cdot 1)$ & 0.003 \\
\hline
\end{tabular}

IR_FGRE, segmented inversion recovery 2D fast gradient echo; SS_SSFP, single-shot inversion recovery 2D steady-state free precession.

were more frequent in cine-MR than in LGE imaging, as shown from score $3.5 \pm 0.5$ for cine compared with $4.0 \pm 0.8$ for IR_FGRE and $4.5 \pm 0.4$ for SS_SSFP. Overall image quality in cine was $3 \cdot 8 \pm 0 \cdot 5$, in IR_FGRE $3 \cdot 3 \pm 0 \cdot 7$ and in SS_SSFP $3.8 \pm 0 \cdot 4$. With cine-MRI in general, there were more artefacts in the basal segments of the left ventricle compared with middle and apical segments, Fig. 1.

The two observers achieved fair agreement of the quality assessment in three of the four parameters (kappa 0.41-0.60) for IR_FGRE, while in SS_SSFP, the parameters showed generally lower agreement according to the kappa statistics.

\section{Myocardial volume, infarct size and global systolic performance}

Myocardial volume was 7\% higher using SS_SSFP (171 ml) compared with IR_FGRE $(159 \mathrm{ml}, \mathrm{P}<0.001)$. No differences were found for infarct size or extent, Table 3. Figure 2 depicts the line of regression of the myocardial volume, infarct volume and infarct extent for the two methods with corresponding Bland-Altman diagram.

The methodological error ( $\left.S_{\text {method }}\right)$ of myocardial volume and infarct size revealed no difference between the two methods, but a higher error was found in the determination of end diastolic volume, Table 4.

The correlations of WMSI on infarct volume (all segments were successfully visualized) were not significantly different for the two methods, and the regression coefficients (slopes) were almost identical (WMSI $=1.27+0.020 x$ IR_FGRE_inf $(\%)$; $\mathrm{r}=0.52 ; \quad \mathrm{P}=0.02$ and WMSI $=1.25+0.024 x$ SS_SSFP_inf $(\%) ; r=0.54 ; P=0.01$; i.e. the slope difference between the two methods was not statistically significant, $(P>0 \cdot 9)$. Left ventricular ejection fraction, determined with echocardiography, was $39 \cdot 3 \pm 8 \cdot 6 \%$, Table 1 .

The segmented reference sequence took on an average of $8.8 \pm 2.0 \mathrm{~min}$ to acquire short and long axis images, while the

Figure 1 Examples of improved myocardial delineation and artefact reduction using the fast sequence. Left panel: LGE image, upper and lower from two different patients, acquired with the segmented IR_FGRE sequence. White myocardium is scar, black is healthy myocardium. Right panel: LGE image, corresponding slice acquired with the SS SSFP sequence. Note the improved delineation of the myocardium and infarct (upper row) and the reduction of artefacts (lower row) on the SS_SSFP slices. IR_FGRE, segmented inversion recovery 2D fast gradient echo; LGE, late gadolinium enhancement; SS_SSFP, single shot inversion recovery 2D steady-state free precession.

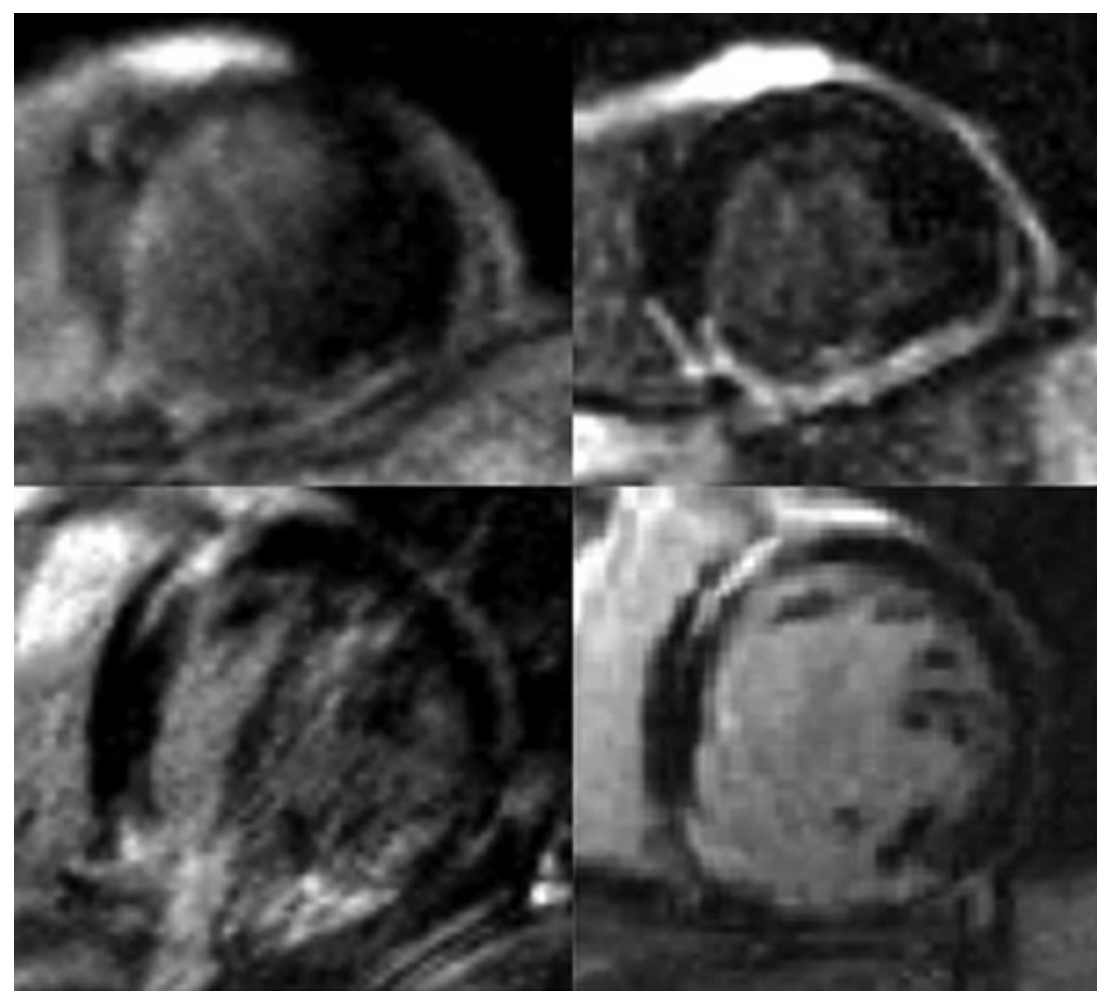


Table 3 Left ventricular measurements and scar size. Mean values (two interpreters) and SD calculated for EDV, myo volume, inf volume, inf extent and the difference between them. Statistically significant differences are bolded.

\begin{tabular}{|c|c|c|c|c|c|c|c|}
\hline \multirow[b]{2}{*}{ Interpreter } & \multirow[b]{2}{*}{ Parameter } & \multicolumn{2}{|c|}{ IR_FGRE } & \multicolumn{2}{|c|}{ SS_SSFP } & \multirow[b]{2}{*}{ Diff* } & \multirow[b]{2}{*}{$P$-value } \\
\hline & & Mean & SD & Mean & SD & & \\
\hline \multirow[t]{4}{*}{ Interpreter 1} & EDV (ml) & $178 \cdot 0$ & $59 \cdot 3$ & $187 \cdot 9$ & $60 \cdot 7$ & $-9 \cdot 9$ & $0 \cdot 233$ \\
\hline & Myo_vol (ml) & $136 \cdot 3$ & $23 \cdot 0$ & $150 \cdot 8$ & $27 \cdot 8$ & $-14 \cdot 5$ & $<0.001$ \\
\hline & Inf_vol (ml) & $18 \cdot 3$ & $14 \cdot 6$ & $16 \cdot 4$ & $13 \cdot 6$ & 1.9 & 0.599 \\
\hline & Inf_extent (\%) & $12 \cdot 9$ & $9 \cdot 4$ & $10 \cdot 7$ & $8 \cdot 0$ & $2 \cdot 2$ & $0 \cdot 210$ \\
\hline \multirow[t]{4}{*}{ Interpreter 2} & $\overline{\mathrm{EDV}}(\mathrm{ml})$ & $171 \cdot 4$ & $51 \cdot 6$ & $161 \cdot 6$ & $51 \cdot 6$ & $9 \cdot 8$ & 0.090 \\
\hline & Myo_vol (ml) & $182 \cdot 2$ & $36 \cdot 0$ & $190 \cdot 6$ & $34 \cdot 3$ & $-8 \cdot 4$ & 0.052 \\
\hline & Inf_vol (ml) & $22 \cdot 5$ & $18 \cdot 2$ & $22 \cdot 2$ & $17 \cdot 0$ & $0 \cdot 3$ & $0 \cdot 498$ \\
\hline & Inf_extent (\%) & $11 \cdot 9$ & $8 \cdot 4$ & $11 \cdot 3$ & $7 \cdot 3$ & $0 \cdot 7$ & $0 \cdot 466$ \\
\hline \multirow[t]{4}{*}{ Mean of 1 and 2} & EDV (ml) & $174 \cdot 7$ & $54 \cdot 9$ & $174 \cdot 8$ & $55 \cdot 1$ & $-0 \cdot 1$ & 0.994 \\
\hline & Myo_vol (ml) & $159 \cdot 2$ & $28 \cdot 0$ & $170 \cdot 7$ & $29 \cdot 6$ & $-11 \cdot 4$ & $<0.001$ \\
\hline & Inf_vol (ml) & $20 \cdot 4$ & $15 \cdot 8$ & $19 \cdot 3$ & $14 \cdot 6$ & $1 \cdot 1$ & 0.599 \\
\hline & Inf_extent (\%) & $12 \cdot 4$ & $8 \cdot 6$ & $11 \cdot 0$ & $7 \cdot 3$ & $1 \cdot 4$ & 0.229 \\
\hline
\end{tabular}

IR_FGRE, segmented inversion recovery 2D fast gradient echo; SS_SSFP, single-shot inversion recovery 2D steady-state free precession; EDV, end diastolic volume; Myo, myocardial; Inf, infarct.

*Difference IR_FGRE - SS_SSFP.

corresponding single shot acquisition took $4.4 \pm 1.6 \mathrm{~min}$, $(\mathrm{P}<0.001)$. Patient's heart rate was $71 \pm 19$ beats $\mathrm{min}^{-1}$ during scanning with the IR_FGRE sequence and $73 \pm 17$ beats min $^{-1}$ during the SS_SSFP sequence, $P=$ n.s.

\section{Signal-to-noise ratio and contrast-to-noise ratio}

SNR was higher (better) in IR_FGRE compared to SS_SSFP, but this difference was statistically significant only for scar and for the bp, Table 5. No significant difference was found between the two methods regarding CNR.

\section{Discussion}

One way of circumventing the problem of motion artefacts in cardiac MRI is to sacrifice spatial resolution for an improved temporal resolution. This can be achieved by limiting read-out to central k-space as in SS_SSFP. Few studies have been published on the effect of these adjustments compared to the conventional LGE sequence (segmented gradient echo). Comparisons have almost always been performed on patients in sinus rhythm (Huber et al., 2004, 2006; Li et al., 2004).

\section{Quality assessment}

We found that SS_SSFP produced images with fewer artefacts than IR_FGRE. Segmentation of the myocardium as well as of the infarct area was facilitated. Overall, the fast sequence was favourably rated compared with the segmented sequence, but infarct volume and infarct extent did not differ significantly between the two methods. This is in line with the results from $\mathrm{Li}$ et al. (2004) who, in patients with sinus rhythm, observed less motion artefacts with SS_SSFP compared to IR_FGRE, which, however, did not influence the result of the infarct size determination. Their average score for image quality was almost identical for the two sequences.

In our study, ongoing atrial fibrillation was expected to influence cine as well as still images. Cine images were reconstructed as an average heart beat with input from RRintervals of different cycle lengths introducing significant motion into the diastolic phase. This resulted in a quality rating that was worse for cine than for any of the two still sequences. Because of significant motion blurring on cine-MRI, wall motion had to be assessed on real-time echocardiographic loops acquired during $3 \mathrm{~s}$, because short cycle lengths allow for less ventricular filling and wall motion may appear reduced in the following beat. Assessment of several heart beats is thus necessary to allow a visual compensation for abnormalities induced by variable filling. This difficulty in wall motion assessment by MRI is a serious limitation if scar imaging is used to define viability in patients with atrial fibrillation, but could be overcome in newer MRI scanners with the availability of realtime cine imaging (Barkhausen et al., 2002; Hori et al., 2003). Single shot imaging displayed superior image quality in all aspects evaluated compared with segmented imaging. However, according to the kappa statistical analysis of the quality assessment, the agreement between the two observers was lower for SS_SSFP than for IR_FGRE, even if the absolute difference in values between the two observers often represented only one step. Thus, it may appear contradictory that an improvement in image quality caused a larger spread in ratings between the two observers. This fact shows the difficulty in quantifying what is basically a qualitative parameter.

When acquiring the fast sequence, the technician followed the motion of the respiration and could initiate the sequence in end-expiration, without the need to interact with the patient. This speeded up the acquisition time, halving it to 4.5 instead of 9 min. 

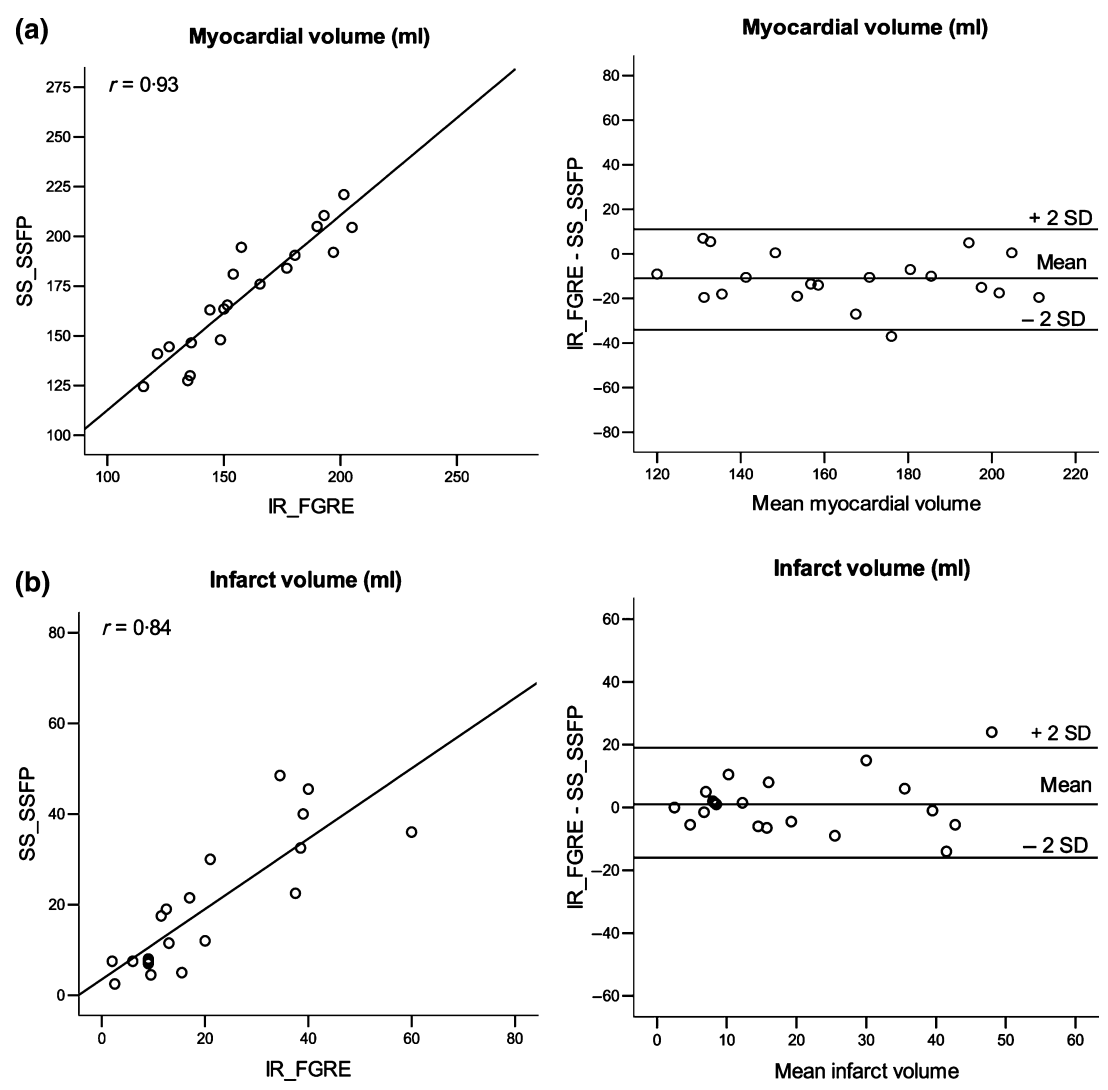

Figure 2 Comparison of myocardial volume and infarct size between IR_FGRE and SS_SSFP. Left: Determination of left ventricular myocardial volume (a), infarct volume (b) and infarct extent (c), IR_FGRE versus SS_SSFP. Regression line is shown. Right: corresponding BlandAltman plot. Values are averaged from two observers. IR_FGRE, segmented inversion recovery 2D fast gradient echo; SS_SSFP, single shot inversion recovery 2D steady-state free precession.

(c)

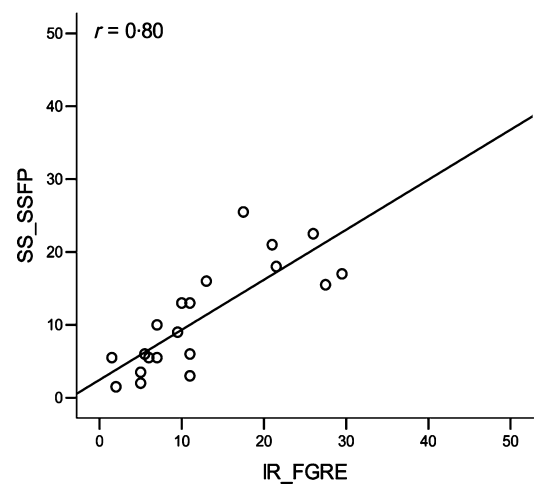

Infarct extent (\%)

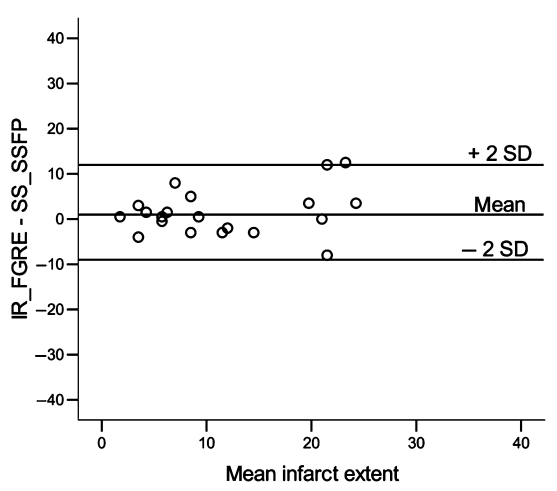

\section{Infarct size and wall motion}

Despite the improvement in image quality from using single shot imaging, there was no difference in infarct size between the two methods, even if myocardial volume was somewhat larger with single shot imaging. This could be because of generally high SNR and CNR for the infarct area using any of the two techniques, making infarct delineation rather straight forward, as previously found in sinus rhythm (Huber et al., 2004, 2006; Li et al., 2004). However, for individual patients with thin subendocardial scar, it appears essential that motion artefact should be suppressed to allow a correct diagnosis. The correlation between wall motion and infarct size was moderate and not different between the two methods. A similar correlation of WMSI on autopsy determined infarct size has been reported by Shen et al. (1991).

\section{Measurements of signal intensity and contrast}

To acquire the functional information needed for cardiac MR imaging, a high SNR and/or CNR is very important (Wen et al., 1997). A high SNR is important to allow parallel imaging and thus reduces scanning time or increases spatial resolution. High CNR is important for contour detection and for the detection of smaller cardiac structures in general. A lower SNR and CNR could be expected with the fast sequence because of higher noise values. In our study, we found a significant difference in $\mathrm{SNR}_{\text {inf }}$ and $\mathrm{SNR}_{\mathrm{bp}}$, however, the magnitude of the difference 
Table 4 Methodological error in absolute numbers and per cent of mean. Statistically significant differences are bolded.

\begin{tabular}{lccccccc}
\hline & \multicolumn{2}{c}{ IR_FGRE } & & \multicolumn{2}{c}{ SS_SSFP } & \\
\cline { 2 - 3 } Parameter & Absolute & Per cent & & Absolute & Per cent & & P-value \\
\hline EDV (ml) & $12 \cdot 5$ & $7 \cdot 2$ & & $24 \cdot 5$ & $14 \cdot 0$ & $\mathbf{0} \cdot 003$ \\
Myo_vol (ml) & $36 \cdot 1$ & $22 \cdot 7$ & & $31 \cdot 3$ & $18 \cdot 4$ & $0 \cdot 730$ \\
Inf_vol (ml) & $7 \cdot 4$ & $36 \cdot 4$ & & $7 \cdot 8$ & $40 \cdot 2$ & $0 \cdot 422$ \\
Inf extent $(\%)$ & $3 \cdot 4$ & $27 \cdot 3$ & & $3 \cdot 1$ & $28 \cdot 3$ & 0.642 \\
\hline
\end{tabular}

IR_FGRE, segmented inversion recovery 2D fast gradient echo; SS_SSFP, single-shot inversion recovery 2D steady-state free precession; EDV, end diastolic volume; Myo, myocardial; Inf, infarct.

Table 5 SI, SNR and CNR as determined from IR_FGRE and SS_SSFP. Mean values and $\mathrm{SD}$ of SI, SNR and CNR. $\mathrm{SI}_{\mathrm{myo}}, \mathrm{SI}_{\mathrm{inf}}, \mathrm{SI}_{\mathrm{bp}}$ and air refer to normal myocardium, infarcted myocardium, bp inside the left ventricle and air outside the patient. Noise is defined as $\mathrm{SI}_{\text {air }}$. SNR and CNR are defined according to article text. Statistically significant differences are bolded.

\begin{tabular}{|c|c|c|c|c|c|c|c|}
\hline \multirow[b]{2}{*}{ Parameter } & \multirow[b]{2}{*}{ Tissue } & \multicolumn{2}{|c|}{ IR_FGRE } & \multicolumn{2}{|c|}{ SS_SSFP } & \multirow[b]{2}{*}{ Diff* I } & \multirow[b]{2}{*}{$P$-value } \\
\hline & & Mean & SD & Mean & SD & & \\
\hline Noise & & 1.5 & 0.6 & $2 \cdot 7$ & 0.6 & $-1 \cdot 1$ & $<0.001$ \\
\hline \multirow[t]{3}{*}{ SI } & Myocardium & $8 \cdot 5$ & $4 \cdot 2$ & $11 \cdot 0$ & $3 \cdot 0$ & $-2 \cdot 5$ & 0.028 \\
\hline & Infarction & $44 \cdot 0$ & $17 \cdot 2$ & $66 \cdot 9$ & $32 \cdot 6$ & $-22 \cdot 9$ & $<0.001$ \\
\hline & Blood & $30 \cdot 8$ & $11 \cdot 9$ & $42 \cdot 6$ & $17 \cdot 0$ & $-11 \cdot 8$ & $<0.001$ \\
\hline \multirow[t]{3}{*}{ SNR } & Myocardium & $6 \cdot 7$ & $5 \cdot 7$ & $4 \cdot 3$ & $1 \cdot 2$ & 2.5 & 0.068 \\
\hline & Infarction & $32 \cdot 4$ & $17 \cdot 1$ & $26 \cdot 1$ & $13 \cdot 7$ & $6 \cdot 3$ & 0.048 \\
\hline & Blood & $22 \cdot 6$ & $10 \cdot 9$ & $16 \cdot 6$ & $7 \cdot 3$ & $6 \cdot 0$ & 0.018 \\
\hline \multirow[t]{2}{*}{ CNR } & Inf-myo & $25 \cdot 6$ & $14 \cdot 8$ & $21 \cdot 9$ & $13 \cdot 1$ & $3 \cdot 8$ & 0.093 \\
\hline & Inf-blood & $9 \cdot 7$ & $9 \cdot 2$ & $9 \cdot 5$ & $9 \cdot 4$ & $0 \cdot 3$ & 0.865 \\
\hline
\end{tabular}

IR_FGRE, segmented inversion recovery 2D fast gradient echo; SS_SSFP, single-shot inversion recovery 2D steady-state free precession; CNR, contrast-to-noise ratio; SNR, signal-to-noise ratio; SI, signal intensity; Inf, infarct; bp, blood pool; myo, myocardial.

*Difference IR_FGRE - SS_SSFP.

was small and there was no significant difference in $\mathrm{CNR}_{\text {inf-myo }}$, $\mathrm{CNR}_{\text {inf-bp }}$ and $\mathrm{SNR}_{\text {myo }}$ between the two sequences. Studies of patients in sinus rhythm, such as the one of Li et al. (2004), found a higher $\mathrm{SNR}_{\mathrm{bp}}, \mathrm{SNR}_{\mathrm{myo}}$ and $\mathrm{CNR}_{\mathrm{bp}-\mathrm{myo}}$ for SS_SSFP compared to IR_FGRE. In contrast, Huber et al. (2006) (also patients in regular rhythm) found significantly lower CNR inf-myo and CNR inf-blood for SS_SSFP compared to IR_FGRE.

\section{Limitations}

This study is based on a rather small number of patients which reduces the chance of finding differences between the two imaging sequences that have been investigated. Mean heart rate was similar for the two scar sequences, which suggests that heart rate variability was in the same range for both scans, important for understanding the degree of motion affecting diastolic acquisition. Because IR_FGRE is the standard sequence used for scar visualization in our institution, one explanation for the difference between the two observers regarding the single shot sequence could be less familiarity with the SS_SSFP sequence and its particular artefacts. These are often located outside of the heart and influence the general assessment but to a lesser extent the delineation of the myocardium and scar.

\section{Conclusions}

In an ageing population, myocardial infarction and cardiac arrhythmia is a growing concern. IR_FGRE ('segmented IR turboFLASH') is a robust sequence when assessing infarct size and ventricular function but requires regular cardiac rhythm and breath holding from the patient. We have shown that in patients with atrial fibrillation, SS_SSFP ('single shot IR trueFISP') acquired during free breathing has significantly better image quality than IR_FGRE acquired during breath holding. The infarct size and the error in its determination were equal for both sequences and the examination time was shorter with SS_SSFP.

\section{Acknowledgments}

The authors recognize Lars Lindeberg, MD, and Emma Kramer, technician, for participating in the investigation of the patients. Financial support was obtained from Futurum - the academy for healthcare, Jönköping County Council, FORSS - The Research Council of Southeastern Sweden, the Swedish Heart-Lung foundation, and Linköping Heart Centre.

\section{References}

Barkhausen J, Ruehm SG, Goyen M, Buck T, Laub G, Debatin JF. MR evaluation of ventricular function: true fast imaging with steady-state precession versus fast low-angle shot cine MR imaging: feasibility study. Radiology (2001); 219: 264-269.

Barkhausen J, Goyen M, Ruhm SG, Eggebrecht H, Debatin JF, Ladd ME. Assessment of ventricular function with single breath-hold real-time steady-state free precession cine MR imaging. AJR Am J Roentgenol (2002); 178: 731-735.

Bongartz G, Golding SJ, Jurik AG, Leonardi M, van Meerten E, Geleijns J, Jessen KA, Panzer W, Shrimpton PC, Tose G, Menzel H-G, Schibilla H, Teunen D The 1999 CEC European Guidelines on Quality Criteria for Computed Tomography, EUR 16262 EN (2000). The Luxembourg Office for publication of the European Communities, Brussels, CEC, 1999.

Dahlberg G Statistical Methods for Medical and Biological Students (1940). George Allen \& Unwin Ltd, London.

Gupta A, Lee VS, Chung YC, Babb JS, Simonetti OP. Myocardial infarction: optimization of inversion times at delayed contrastenhanced MR imaging. Radiology (2004); 233: 921-926.

Heiberg E, Ugander M, Engblom H, Gotberg M, Olivecrona GK, Erlinge D, Arheden H. Automated quantification of myocardial infarction from MR images by accounting for partial volume effects: animal, phantom, and human study. Radiology (2008); 246: 581-588.

Hillenbrand HB, Kim RJ, Parker MA, Fieno DS, Judd RM. Early assessment of myocardial salvage by contrast-enhanced magnetic resonance imaging. Circulation (2000); 102: 1678-1683.

Hori Y, Yamada N, Higashi M, Hirai N, Nakatani S. Rapid evaluation of right and left ventricular function and mass using real-time true-FISP 
Image quality and scar size in atrial fibrillation, L. Rosendahl et al. 129

cine MR imaging without breath-hold: comparison with segmented true-FISP cine MR imaging with breath-hold. J Cardiovasc Magn Reson (2003); 5: 439-450.

Huber A, Schonberg SO, Spannagl B, Rieber J, Klauss V, Reiser MF. [Determining myocardial viability in myocardial infarct. Comparison of single and multisclice MRI techniques with TurboFlash and TrueFISP sequences]. Radiologe (2004); 44: 146-151.

Huber A, Schoenberg SO, Spannagl B, Rieber J, Erhard I, Klauss V, Reiser MF. Single-shot inversion recovery TrueFISP for assessment of myocardial infarction. AJR Am J Roentgenol (2006); 186: 627-633.

Kim RJ, Fieno DS, Parrish TB, Harris K, Chen E-L, Simonetti O, Bundy J, Finn JP, Klocke FJ, Judd RM. Relationship of MRI delayed contrast enhancement to irreversible injury, infarct age, and contractile function. Circulation (1999); 100: 1992-2002.

Kim RJ, Wu E, Rafael A, Chen EL, Parker MA, Simonetti O, Klocke FJ, Bonow RO, Judd RM. The use of contrast-enhanced magnetic resonance imaging to identify reversible myocardial dysfunction. $\mathrm{N}$ Engl J Med (2000); 343: 1445-1453.

Kitagawa K, Sakuma H, Hirano T, Okamoto S, Makino K, Takeda K. Acute myocardial infarction: myocardial viability assessement in patients early thereafter - comparison of contrast-enhanced MR imaging with resting 201 TI SPECT. Radiology (2003); 226: 138-144.

Li W, Li BS, Polzin JA, Mai VM, Prasad PV, Edelman RR. Myocardial delayed enhancement imaging using inversion recovery single-shot steady-state free precession: initial experience. J Magn Reson Imaging (2004); 20: 327-330.

Lu B, Mao SS, Zhuang N, Bakhsheshi H, Yamamoto H, Takasu J, Liu SC, Budoff MJ. Coronary artery motion during the cardiac cycle and optimal ECG triggering for coronary artery imaging. Invest Radiol (2001); 36: 250-256.
Mahrholdt H, Wagner A, Holly TA, Elliott MD, Bonow RO, Kim RJ, Judd RM. Reproducibility of chronic infarct size measurement by contrastenhanced magnetic resonance imaging. Circulation (2002); 106: 23222327.

Plein S, Bloomer TN, Ridgway JP, Jones TR, Bainbridge GJ, Sivananthan MU. Steady-state free precession magnetic resonance imaging of the heart: comparison with segmented k-space gradient-echo imaging. J Magn Reson Imaging (2001); 14: 230-236.

Schiller NB, Shah PM, Crawford M, DeMaria A, Devereux R, Feigenbaum H, Gutgesell H, Reichek N, Sahn D, Schnittger I, Silverman N, Tajik J. Recommendations for quantitation of the left ventricle by twodimensional echocardiography. American Society of Echocardiography Committee on Standards, Subcommittee on Quantitation of Two-Dimensional Echocardiograms. J Am Soc Echocardiogr (1989); 2: 358-367.

Shen WK, Khandheria BK, Edwards WD, Oh JK, Miller FA Jr, Naessens JM, Tajik AJ. Value and limitations of two-dimensional echocardiography in predicting myocardial infarct size. Am J Cardiol (1991); 68: 1143-1149.

Simonetti OP, Kim RJ, Fieno DS, Hillenbrand HB, Wu E, Bundy JM, Finn JP, Judd RM. An improved MR imaging technique for the visualization of myocardial infarction. Radiology (2001); 218: 215-223.

Thiele H, Nagel E, Paetsch I, Schnackenburg B, Bornstedt A, Kouwenhoven M, Wahl A, Schuler G, Fleck E. Functional cardiac MR imaging with steady-state free precession (SSFP) significantly improves endocardial border delineation without contrast agents. J Magn Reson Imaging (2001); 14: 362-367.

Wen H, Denison TJ, Singerman RW, Balaban RS. The intrinsic signal-tonoise ratio in human cardiac imaging at 1.5, 3, and 4 T. J Magn Reson (1997); 125: 65-71. 